\title{
Face Recognition using Multiple Face Eigen Subspaces
}

\author{
Aishwarya P \\ Asst. Professor, \\ Department of CSE, \\ Atria Institute of Technology, \\ Anand Nagar, \\ Bangalore - 560024
}

\begin{abstract}
Face recognition has been widely explored in the past years. A lot of techniques have been applied in various applications. Robustness and reliability have become more and more important for these applications especially in security systems. In this paper, a variety of approaches for face recognition are reviewed first. These approaches are classified according to three basic tasks: face representation, face detection, and face identification. An implementation of the appearance-based face recognition method, the eigenface recognition approach, is reported. This method utilizes the idea of the principal component analysis and decomposes face images into a small set of characteristic feature images called eigenfaces.

Our experiments strongly supports the proposed area in which an effective performance over the traditional "eigenface" has been observed when tested on the same face base.

The idea of using eigenfaces was partially motivated by the work of Sirovich and Kirby [2] for efficiently representing pictures of faces using principal component analysis. Starting with an ensemble of original face images, they calculated a best coordinate system for image compression, where each coordinate is actually an image which they termed an eigenpicture. They argued that, at least in principle, any collection of face images can be approximately reconstructed by storing a small collection of weights for each face and a small set of standard pictures (the eigenpictures). The weights describing each face are found by projecting the face image onto each eigen picture.
\end{abstract}

The full text of the article is not available in the cache. Kindly refer the IJCA digital library at www.ijcaonline.org for the complete article. In case, you face problems while downloading the full-text, please send a mail to editor at editor@ijcaonline.org 